\title{
Fatores associados à intenção de carreira na atenção primária à saúde entre estudantes de Medicina
}

\section{Factors associated with career intention in primary health care among medical students}

\author{
Camila Zamban de Miranda' (D) | camilazamban@gmail.com

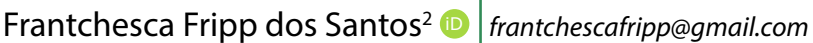 \\ Karina Cenci Pertile ${ }^{1}$ (D) kapertile@gmail.com \\ Simone de Melo Costa ${ }^{2}$ (D) smelocosta@gmail.com \\ Antônio Prates Caldeira² (1) antonio.caldeira@unimontes.br \\ Mariane Silveira Barbosa² (D) msb.mariane@gmail.com
}

\begin{abstract}
RESUMO
Introdução: A reorganização da assistência à saúde a partir da criação do Sistema Único de Saúde (SUS) tem sido orientada por uma proposta de expansão e fortalecimento da atenção primária à saúde (APS). Nesse cenário, a escassez de profissionais capacitados é considerado um dos principais desafios para a consolidação da APS. Dentre as possibilidades de carreira após a conclusão da graduação, a maioria dos médicos optam por buscar especialidades focais para atuar em outros níveis de atenção.

Objetivo: Assim, este artigo, de natureza quantitativa, se propõe a analisar a prevalência e os fatores associados ao interesse de estudantes de Medicina em seguir carreira na APS.

Método: Foi realizado um estudo transversal, com estudantes de Medicina dos dois últimos anos do curso, em quatro escolas médicas do estado de Minas Gerais, no Brasil. Participaram 524 estudantes que responderam a um questionário autoaplicado, elaborado pelos autores, com questões que incluíam os perfis sociodemográfico e econômico do estudante, a interação com a APS ao longo curso, a intenção de carreira e o desejo de cursar Residência em Medicina de Família e Comunidade (RMFC).

Resultado: Os resultados evidenciaram que $26,3 \%$ dos entrevistados referiram interesse em seguir carreira na APS, percentual mais alto que o registrado em estudos anteriores. Quanto ao fato de a APS ser uma opção de trabalho temporária, 79,3\% dos participantes referiram que pretendem atuar nesse campo após o término da graduação. Dez por cento dos estudantes informaram que pretendem cursar RMFC. Entre os fatores associados à intenção de atuar na APS, destaca-se a vivência de uma experiência exitosa na APS ao longo da graduação.

Conclusão: Os resultados devem ser considerados por gestores da área da saúde e da educação médica, fomentando estratégias que propiciem experiências exitosas na APS, com maior incentivo à integração ensino-serviço e à inserção de estudantes, ao longo da graduação, em uma rede de APS fortalecida. Iniciativas como ampliação de carga horária na APS durante a graduação, aumento do número de professores especialistas em MFC e qualificação da rede de saúde dos municípios onde essas instituições de ensino superior estão inseridas podem ser potentes no sentido de aumentar o interesse dos formandos de atuar na APS.
\end{abstract}

Palavras-chave: Estudantes de Medicina; Atenção Primária à Saúde; Escolha da Profissão.

\section{ABSTRACT}

Introduction: Health assistance reorganization has been oriented, since the Unified Health System (SUS) creation, by a proposal of expansion and strengthening of Primary Health Care (PHC). In this context, the scarcity of trained professionals is considered one of the main challenges for PHC consolidation. Among the career options after graduation, most physicians choose to seek focal specializations to work in other health care levels.

Objective: Therefore, this quantitative study aims to analyze the prevalence and associated factors of medical students' interest in following a career in PHC.

Method: A cross-sectional study was conducted with medical students attending the last two years in four medical schools in the state of Minas Gerais, Brazil. A total of 524 students participated by answering a self-administered questionnaire, which was prepared by the authors, including questions regarding sociodemographic and economic profile, interaction with PHC during undergraduate school, career intention and intention to attend the Family and Community Medicine Residency (FCMR) program.

Results: The results showed that $26.3 \%$ of the respondents indicated interest in following a career in PHC, a higher percentage when compared to previous studies. Regarding PHC being a temporary work choice, 79,3\% of the participants indicated that they intend to work in this field after graduation. Ten percent of the students informed they intend to attend the FCMR program. Among the factors associated with the intention to work in PHC, a successful PHC experience during undergraduate school stands out.

Conclusion: The results should be taken into consideration by health and medical education managers, fostering strategies that promote successful PHC experiences, encouraging learning-service integration and including students in a consolidated PHC network during undergraduate school. Initiatives such as increasing the workload in PHC during undergraduate school, increasing the number of FMC specialized teachers and health network improvement in the municipalities where these Higher Education Institutions are located can be powerful actions toward increasing the graduating students' interest to work in PHC.

Keywords: Medical Students; Primary Health Care; Career Choice.

${ }^{1}$ Universidade Federal dos Vales do Jequitinhonha e Mucuri, Diamantina, Minas Gerais, Brasil.

2 Universidade Estadual de Montes Claros, Montes Claros, Minas Gerais, Brasil.

Editora-chefe: Rosiane Viana Zuza Diniz. | Editora associada: Rosana Alves.

Recebido em 20/09/20; Aceito em 12/05/21. | Avaliado pelo processo de double blind review. 


\section{INTRODUÇÃO}

A reorganização da assistência à saúde a partir da criação do Sistema Único de Saúde (SUS) tem sido orientada por uma proposta de expansão e fortalecimento da atenção primária à saúde (APS). Esse processo assumiu particularidade no Brasil com a implementação de equipes multiprofissionais de saúde compostas por médico generalista, enfermeiro, técnico de enfermagem e agentes comunitários de saúde, que são responsáveis pelo cuidado integral de uma determinada população, modelo chamado de Estratégia Saúde da Família (ESF), que vem se consolidando como principal estratégia de organização da APS brasileira'.

A escassez de médicos para atuar nas equipes da ESF é considerada um dos principais desafios para a consolidação da APS no Brasil2-4. Além da falta de médicos, esses profissionais estão frequentemente mal distribuídos geograficamente, concentrados em cidades de maior porte populacional, localizadas em regiões economicamente mais desenvolvidas 4 . Todavia, essa não é uma situação peculiar do Brasil, pois outros países também registram dificuldades de provimento de médicos no âmbito da APS ${ }^{5-8}$.

Alguns países tomaram medidas para aumentar as vagas de treinamento para médicos generalistas, como o Canadá que dobrou as vagas para treinamento em medicina de família entre 2000 e 2013, como parte de um esforço para aumentar o acesso à atenção primária. Entretanto, na maioria dos países da Organização para a Cooperação e Desenvolvimento Econômico (OCDE), os médicos especialistas ganham mais que os médicos generalistas, o que, dessa forma, incentiva financeiramente que os médicos se especializem

No Brasil, diversas propostas de intervenção nos processos de formação de recursos humanos em saúde têm sido reconhecidas como indutoras de uma nova missão social das escolas médicas nos cursos de graduação e pós-graduação ${ }^{10}$. Em 2014, o Ministério da Educação instituiu as novas Diretrizes Curriculares Nacionais (DCN) para os cursos de Medicina que reforçam a necessidade de formação de profissional médico para as necessidades da população e recomendam a inserção do estudante nas redes de serviços de saúde ao longo de todo o curso de graduação. Para uma inserção mais oportuna dos estudantes na APS, houve necessidade de maior aproximação entre as escolas médicas e os serviços de saúde ${ }^{11}$, e o governo federal passou a adotar, a partir desse momento, políticas indutoras que propiciam a integração ensino-serviço, colocando em prática programas de incentivo às mudanças curriculares ${ }^{12}$.

Outras iniciativas importantes em relação à formação e ao provimento de médicos para o Brasil foram o Programa Mais Médicos (PMM) e o incentivo à ampliação de vagas de Residência em Medicina de Família e Comunidade (RMFC). O PMM foi criado em 2013 com o objetivo de aumentar a densidade de médicos no Brasil a partir de três ações principais: ampliação de vagas em cursos de graduação, aumento de vagas de residência médica nas áreas prioritárias para o SUS e provimento de médicos na atenção básica, na periferia das grandes cidades e nos interiores dos estados ${ }^{13}$. Um estudo recente de avaliação do impacto do PMM registrou um substancial aumento na oferta de médicos em APS, o que contribuiu para reduzir o número de municípios com escassez desses profissionais ${ }^{14}$.

Apesar das iniciativas em prover recursos humanos em áreas prioritárias para os sistemas de saúde, há uma incongruência em relação às necessidades de formação médica e às escolhas individuais de carreira dos profissionais médicos não só no Brasil, mas também em vários países ${ }^{15}$, indicando que, ao mesmo tempo que se busca o redirecionamento dos sistemas de saúde, em alguns países observa-se o declínio na escolha de carreiras ligadas à APS ${ }^{16}$.

Nos últimos anos, a abertura de novas escolas médicas e o destaque alcançado pelo PMM definiram um novo cenário, com valorização do espaço de práticas na atenção primária. Nesse sentido, o presente estudo teve como objetivo identificar a prevalência e os fatores associados ao interesse de estudantes de Medicina em seguir carreira profissional na APS.

\section{MÉTODOS}

Trata-se de um estudo transversal, analítico, realizado com a participação de estudantes de Medicina dos últimos dois anos do curso de graduação em Medicina, em quatro instituições de ensino superior (IES) do estado de Minas Gerais, no Brasil. O estado possui 46 escolas médicas, e, considerando a dificuldade logística de abordagem amostral para alcançar representatividade necessária entre todas as instituições, optou-se por uma amostragem de conveniência, em que se selecionaram quatro IES, sendo uma na capital e três no interior do estado, com entrevista de todos os estudantes regularmente matriculados no quinto ou no sexto ano. O instrumento de coleta de dados foi um questionário autoaplicado, elaborado pelos autores a partir dos objetivos do estudo, com questões que incluíam os perfis sociodemográfico e econômico do estudante, a interação com a APS ao longo curso, a intenção de carreira e o desejo de cursar RMFC. Após elaboração do instrumento, foi realizada a validação de face e de conteúdo por seis experts na área, além de um estudo-piloto com estudantes do quinto ano de outra faculdade de Medicina do estado de Minas Gerais, não incluída no estudo.

Aplicou-se o questionário em formato físico e em meio virtual. O contato presencial com os estudantes foi realizado por estudantes de iniciação científica, após apresentação do projeto às IES pelos pesquisadores. A equipe de coleta de 
dados foi especialmente treinada e visitava periodicamente as IES selecionadas, identificando e abordando estudantes dos últimos períodos do curso. Fizeram-se diversas visitas às IES com o objetivo de alcançar um número maior de estudantes. Após três visitas a cada turma ou subturma, consideraramse perdas, independentemente do motivo alegado, aqueles estudantes que não aceitaram participar da pesquisa ou que não foram encontrados.

Para ampliar o número de participantes, solicitou-se às IES uma lista de contatos eletrônicos dos estudantes, e o instrumento de coleta de dados em formato digital foi enviado por e-mail para eles, com uma breve explicação da pesquisa. Consideraram-se elegíveis para o estudo todos os estudantes com idade igual ou superior a 18 anos, regularmente matriculados no quinto ou no sexto ano das escolas de Medicina pesquisadas. Não se definiram critérios de exclusão.

Todos os dados foram submetidos ao tratamento estatístico no programa IBM SPSS versão 22.0. Realizou-se a análise estatística descritiva com cálculo de proporções e análise bivariada utilizando o teste qui-quadrado de Pearson. As variáveis que se mostraram estatisticamente associadas com o desfecho avaliado ("interesse em seguir carreira profissional na APS") até o nível de $20 \%(p \leq 0,20)$ foram avaliadas de forma conjunta por meio da regressão logística. Para o modelo final, considerou-se o nível de significância de $5 \%(p<0,05)$, e inseriram-se os valores de odds ratio (OR) e seus respectivos intervalos de confiança de 95\% (IC95\%).

A presente pesquisa foi aprovada pelo Comitê de Ética em Pesquisa envolvendo seres humanos da Universidade Estadual de Montes Claros (Unimontes) de Minas Gerais, em respeito à Resolução n 466/2012 do Conselho Nacional de Saúde do Ministério da Saúde. Foi garantido o anonimato das informações prestadas pelos estudantes, e todos os participantes assinaram um Termo de Consentimento Livre e Esclarecido. O projeto desta pesquisa foi aprovado pelo Comitê de Ética em Pesquisa de uma das instituições participantes do estudo - Parecer n 2.426.972.

\section{RESULTADOS}

Participaram deste estudo 524 estudantes, constituindo uma taxa de resposta de $45 \%$ da população eletiva para o estudo. A maioria dos entrevistados era do sexo feminino, entre 21 e 25 anos, solteira e autorreferida branca. Mais da metade cursou ensino médio em escolas privadas e informou pais graduados. Essas e outras características do grupo são apresentadas na Tabela 1.

Houve maior participação entre os estudantes do sexto ano do curso de Medicina, composto pelos $11^{\circ} \mathrm{e} 12^{\circ}$ períodos do curso. Pouco mais da metade $(55,5 \%)$ dos entrevistados
Tabela 1. Caracterização do perfil sociodemográfico de estudantes dos últimos dois anos do curso de Medicina, Minas Gerais, Brasil.

\begin{tabular}{llll}
\hline & Variável & $\mathbf{n}$ & $\%$ \\
\hline Idade (anos) & & & \\
& $\leq 25$ & 349 & 66,7 \\
& $>25$ & 174 & 33,3
\end{tabular}

Sexo

\begin{tabular}{lll} 
Feminino & 298 & 57,0 \\
Masculino & 225 & 43,0 \\
\hline
\end{tabular}

Estado civil

\begin{tabular}{ccc} 
Solteiro & 480 & 91,8 \\
Casado/união estável & 40 & 7,6 \\
\hline
\end{tabular}

Cor da pele (autorreferida)

\begin{tabular}{ccc} 
Branca & 277 & 54,7 \\
Amarela & 8 & 1,6 \\
Parda & 194 & 38,3 \\
Preta & 27 & 5,3 \\
\hline
\end{tabular}

Tipo de escola em que cursou o ensino médio

Pública

$108 \quad 20,7$

Privada

41579,3

Escolaridade do pai

$\begin{array}{lll}\text { Nenhuma } & 1 & 0,2\end{array}$

Ensino fundamental $\quad 79 \quad 15,2$

Ensino médio $\quad 169 \quad 32,6$

Ensino superior ou pós-graduação $\quad 270 \quad 52,0$

Escolaridade da mãe

\begin{tabular}{ccc} 
Ensino fundamental & 32 & 6,1 \\
Ensino médio & 121 & 23,1 \\
superior ou pós-graduação & 370 & 70,8 \\
\hline
\end{tabular}

Ensino superior ou pós-graduação

Possui graduação anterior em outro curso

Sim $54 \quad 10,3$

Não $\quad 469 \quad 89,7$

Tipo de IES*

\begin{tabular}{lll} 
Pública & 321 & 61,3 \\
Privada & 202 & 38,7 \\
\hline
\end{tabular}

IES situada no interior/na capital

Interior

35567,9

Capital

$168 \quad 32,1$

Forma de admissão na IES

$$
\text { Prouni** }
$$

Enem***/ vestibular

47691,5

Transferência interna

$9 \quad 1,7$

*IES: instituição de ensino superior; **Prouni: Programa Universidade para Todos; ***Enem: Exame Nacional do Ensino Médio. 
referiu já ter escolhido em qual área quer se especializar após a conclusão do curso, dos quais 58,3\% reportaram ter maior aptidão para especialidades clínicas (Tabela 2). A maior parte $(78,5 \%)$ dos entrevistados referiu ter vivenciado experiência positiva na APS durante a graduação. Mais da metade dos alunos participam de pelo menos uma liga acadêmica, dos quais apenas 4,8\% ( $n=14)$ atuam em ligas acadêmicas relacionadas à MFC. Quanto ao fato de a APS ser uma opção de trabalho, ainda que temporária, $79,3 \%$ dos participantes referiram que

Tabela 2. Caracterização do perfil acadêmico de estudantes dos últimos dois anos do curso de Medicina, Minas Gerais, Brasil.

\begin{tabular}{ccc}
\hline Variável & $\mathbf{n}$ & $\%$ \\
\hline Semestre da graduação que está cursando & & \\
$9^{\circ}$ & 93 & 18,0 \\
$10^{\circ}$ & 87 & 16,8 \\
$11^{\circ}$ & 221 & 42,7 \\
$12^{\circ}$ & 117 & 22,6 \\
\hline
\end{tabular}

Já escolheu qual especialidade quer cursar?

\begin{tabular}{lll} 
Sim & 289 & 55,5 \\
Não & 232 & 44,5 \\
\hline
\end{tabular}

Maior aptidão para especialidades clínicas ou cirúrgicas?

\begin{tabular}{ccc} 
Clínica & 304 & 58,3 \\
Cirúrgica & 164 & 31,4 \\
Não sei & 54 & 10,3 \\
\hline
\end{tabular}

Experiência na APS $^{* *}$ durante a graduação

\begin{tabular}{ccc} 
Ótima/boa & 408 & 78,5 \\
Regular & 92 & 17,7 \\
Ruim/péssima & 20 & 3,8 \\
\hline
\end{tabular}

Participa/participou de ligas acadêmicas?

\begin{tabular}{lll} 
Sim & 290 & 55,6 \\
Não & 232 & 44,4 \\
\hline
\end{tabular}

Interesse em RMFC* após a graduação

\begin{tabular}{ccc} 
Sim & 52 & 10,0 \\
Não & 363 & 69,8 \\
Não sei & 105 & 20,2 \\
\hline
\end{tabular}

Interesse em seguir carreira na APS?

\begin{tabular}{lll} 
Sim & 136 & 26,3 \\
Não & 382 & 73,7 \\
\hline
\end{tabular}

Interesse em trabalhar na APS após a graduação

$\begin{array}{lll}\text { Sim } & 402 & 79,3 \\ \text { Não } & 105 & 20,7\end{array}$

*RMFC: Residência de Medicina de Família e Comunidade; ${ }^{* *}$ APS: atenção primária à saúde. pretendem atuar na APS após o término da graduação, mesmo sem terem cursado RMFC (Tabela 2).

O motivo determinante para os estudantes trabalharem na APS imediatamente após a conclusão do curso foi o fato de ela ser uma opção de trabalho temporária até que entrem em residência médica de outra área (78,6\%). Esse motivo foi seguido pela disponibilidade de vagas no mercado de trabalho $(47,3 \%)$ e pelo fato de terem vivenciado experiências exitosas em APS durante a graduação, sendo este último com menos

Tabela 3. Fatores determinantes na escolha de atuarem ou não na atenção primária à saúde após a graduação para estudantes dos últimos dois anos do curso de Medicina, Minas Gerais, Brasil.

\begin{tabular}{lcc}
\hline \multicolumn{1}{c}{ Fatores determinantes na escolha } & (n) & (\%)* \\
\hline \multicolumn{1}{c}{ Para atuar na APS** após a graduação $(n=402)$} & \\
\hline $\begin{array}{l}\text { Opção de trabalho até ser aprovado em } \\
\text { residência médica de outra área }\end{array}$ & 316 & 78,6 \\
$\begin{array}{l}\text { Mercado de trabalho com grande } \\
\text { disponibilidade de vagas }\end{array}$ & 190 & 47,3 \\
Vivenciei experiência exitosa em APS no currículo & 120 & 29,9 \\
Boa remuneração & 107 & 26,6 \\
Possibilidade de longitudinalidade no cuidado & 99 & 24,6 \\
Possibilidade de concurso público & 92 & 22,9 \\
$\begin{array}{l}\text { Um bom professor/tutor durante a experiência } \\
\text { na APS }\end{array}$ & 82 & 20,4 \\
Compromisso com o SUS*** & 76 & 18,9 \\
Vivenciei experiência exitosa em APS fora do & 32 & 8,0 \\
currículo & 10 & 2,5 \\
\hline Outros
\end{tabular}

Para NÃO atuar na APS após a graduação $(n=105)$

Pretendo cursar residência em especialidade focal sem atuar na APS antes da residência

7066,7

Falta de um plano de carreira

4139,0

Má remuneração

$35 \quad 33,3$

Vivenciei experiência não exitosa em APS no currículo

$24 \quad 22,9$

Carga horária e horário de trabalho (40 horas, 19 18,1 dias por semana)

19

Preferência por atendimentos clínicos pontuais e não longitudinais

$19 \quad 18,1$

Pouco reconhecimento social $12 \quad 11,4$

Sobrecarga de trabalho

$11 \quad 10,4$

Preferência por não atender pessoas de diferentes idades

$9 \quad 8,6$

Mercado de trabalho com poucas vagas

$5 \quad 4,8$

Outros

$11 \quad 10,4$

*O somatório dos percentuais é maior do que $100 \%$ porque os respondentes poderiam assinalar mais de uma resposta. ${ }^{* *}$ APS: atenção primária à saúde. ${ }^{* *}$ SUS: Sistema Único de Saúde. 
poder de influenciar, com 29,9\% dos respondentes. Dos $20,7 \%$ que não consideram a APS como uma opção de trabalho após o término da graduação, $66,7 \%$ referiram que o principal motivo para essa escolha tem a ver com o fato de poderem cursar residência em especialidade focal sem que tenham atuado na APS antes do ingresso na residência. Os outros fatores determinantes mais citados na escolha de não atuarem na APS após a graduação foram a falta de um plano de carreira (39\%) e a má remuneração (33,3\%) (Tabela 3).

Quando questionados acerca da intenção de cursar residência em MFC, $10 \%$ dos estudantes informaram que pretendem cursar essa residência após formados e 20,2\% mencionaram que ainda não sabem se pretendem ou não cursá-la. Acerca da intenção de seguir carreira profissional da APS, 26,3\% dos entrevistados responderam de forma afirmativa.
A Tabela 4 mostra os resultados das análises bivariadas dos fatores associados à intenção de seguir carreira na APS. Após análise múltipla, registrou-se que as variáveis sexo feminino, possuir graduação anterior, ingressar na IES por meio do Programa Universidade para Todos (Prouni), referir maior aptidão para especialidades clínicas e vivenciar experiência positiva na APS durante a graduação permaneceram associadas à maior chance de intenção de carreira na APS. As variáveis idade, estado civil e escolaridade da mãe não estiveram significativamente associadas à intenção de carreira na APS.

\section{DISCUSSÃO}

O percentual de formandos com interesse em seguir carreira na APS observado neste estudo é próximo ao encontrado em diversos países, e em alguns, como Estados Unidos, Alemanha e França, a intenção de atuar na APS é

Tabela 4. Fatores associados à intenção de seguir carreira na atenção primária à saúde após a graduação para estudantes dos últimos dois anos do curso de Medicina, Minas Gerais, Brasil.

\begin{tabular}{|c|c|c|c|c|c|c|c|}
\hline \multirow{3}{*}{ Variável } & \multicolumn{4}{|c|}{ Interesse em carreira na APS } & \multirow[t]{3}{*}{ p valor ${ }^{1}$} & \multirow[t]{3}{*}{ p valor ${ }^{2}$} & \multirow[t]{2}{*}{ OR (IC95\%)* } \\
\hline & \multicolumn{2}{|c|}{ Sim } & \multicolumn{2}{|c|}{ Não } & & & \\
\hline & $\mathbf{n}$ & $\%$ & $\mathbf{n}$ & $\%$ & & & \\
\hline Sexo & & & & & $<0,001$ & $<0,001$ & \\
\hline Feminino & 100 & 33,7 & 197 & 66,3 & & & $2,30(1,45-3,65)$ \\
\hline Masculino & 36 & 16,3 & 185 & 83,7 & & & 1,00 \\
\hline Modalidade de ingresso & & & & & 0,101 & 0,027 & \\
\hline Prouni & 13 & 37,1 & 22 & 62,9 & & & $2,40(1,11-5,20)$ \\
\hline Enem/vestibular & 126 & 24,6 & 335 & 75,4 & & & 1,00 \\
\hline Possui graduação anterior & & & & & 0,003 & 0,010 & \\
\hline Sim & 23 & 43,4 & 30 & 56,6 & & & $2,51(1,25-5,04)$ \\
\hline Não & 113 & 24,3 & 352 & 75,7 & & & 1,00 \\
\hline Possui maior aptidão para especialidades: & & & & & $<0,001$ & $<0,001$ & \\
\hline Clínicas & 109 & 36,1 & 193 & 63,9 & & & $2,95(1,81-4,80)$ \\
\hline Cirúrgicas/não sei & 27 & 12,5 & 189 & 87,5 & & & 1,00 \\
\hline Experiência na APS durante a graduação & & & & & 0,003 & 0,017 & \\
\hline Ótima/boa & 119 & 29,3 & 287 & 70,7 & & & $2,07(1,14-3,77)$ \\
\hline Regular/ruim/péssima & 17 & 15,2 & 95 & 84,8 & & & 1,00 \\
\hline Idade (anos) & & & & & 0,019 & 0,404 & \\
\hline$>25$ & 56 & 32,7 & 115 & 67,3 & & & - \\
\hline$\leq 25$ & 80 & 23,1 & 266 & 76,9 & & & - \\
\hline Estado civil & & & & & 0,092 & 0,919 & \\
\hline Casado/união estável & 15 & 37,5 & 25 & 62,5 & & & - \\
\hline Solteiro & 121 & 25,3 & 357 & 74,7 & & & - \\
\hline Escolaridade da mãe & & & & & 0,056 & 0,941 & \\
\hline Até ensino fundamental & 13 & 40,6 & 19 & 59,4 & & & - \\
\hline Ensino médio ou superior & 123 & 25,3 & 363 & 74,7 & & & - \\
\hline
\end{tabular}

1 Análise bruta (teste qui-quadrado); 2análise ajustada (teste qui-quadrado); *OR: odds ratio; IC95\%: intervalo de confiança. 
ainda menor. Estudo realizado, no ano de 2019, nos Estados Unidos mostrou que, no país, apenas $14 \%$ dos estudantes de Medicina que ingressam no Programa Nacional de Ingresso em Residências correspondem a residências em atenção primária ${ }^{17}$. Na Alemanha, em 2015, 12\% dos graduandos entrevistados afirmaram que haviam optado por carreira como generalistas da APS (general practitioners - GP) ${ }^{18}$. Na França, outro estudo evidenciou que cerca de $20 \%$ dos estudantes escolheram uma carreira na APS após a graduação ${ }^{19}$. No Paquistão, o resultado foi similar ao presente estudo: em uma pesquisa com 1.400 estudantes de Medicina do último ano, em oito escolas de Medicina, identificou-se que $24,1 \%$ dos entrevistados referiam o interesse em atuar na APS ${ }^{20}$.

Ainda que em alguns países, como o Canadá, haja uma tendência de crescimento da escolha da RMFC, com um aumento na procura que alcançou $38,5 \%$ dos estudantes que selecionaram medicina de família como primeira opção ${ }^{21}$, em todos eles evidencia-se uma falta de alinhamento das necessidades do sistema de saúde com as intenções dos alunos $^{22}$. No Reino Unido, por exemplo, o Departamento de Saúde estimava que, no ano 2013 , seria necessário que $50 \%$ dos graduados se tornassem GP quando, em 2009, apenas $28 \%$ dos graduandos disseram ter intenção de assumir uma posição de trabalho na APS ${ }^{23}$.

No Brasil, um estudo de 2016 realizado na Universidade de São Paulo demonstrou que $47 \%$ dos estudantes do sexto ano de um curso de Medicina tinham a intenção de trabalhar na APS do SUS ${ }^{12}$. Esses resultados já se mostraram bem acima dos encontrados em outro estudo de 2011, no qual apenas $20 \%$ dos graduandos tinham como possibilidade de trabalho a APS após formados ${ }^{24}$. Um estudo de 2012, com mais de mil estudantes de Medicina e médicos brasileiros, identificou que $26 \%$ dos entrevistados optaram por especialidades no grupo de APS, entretanto apenas $1,2 \%$ colocou a medicina de família como primeira escolha de especialização ${ }^{25}$.

Apesar de o desafio de se conseguir força de trabalho adequada para APS ser relatado em vários países, a magnitude e a complexidade do SUS, que é o maior sistema universal de saúde do mundo, fazem com que não existam, no âmbito internacional, dados comparáveis ou países similares ao Brasil. Mesmo assim, esses estudos internacionais demonstram similaridade com o resultado desta pesquisa, com percentuais semelhantes de opção pela APS como carreira, sendo inferior às necessidades dos sistemas de saúde de cada país. Diversas iniciativas têm sido desenvolvidas para enfrentar esse problema. No final de 2014, nos Estados Unidos foi criada a Family Medicine for America's Health (FMAHealth) Workforce Education and Development Tactic Team (WEDTT), com o objetivo de aumentar a porcentagem de estudantes de
Medicina do país que escolhem medicina de família de $12 \%$ a $25 \%$ até o ano 2030, para atender às necessidades do país. $\mathrm{A}$ WEDTT desenvolveu um pacote de ideias de mudança com base em sua teoria do que impulsionará a realização de " 25 x 2030", o que levou a projetos específicos concluídos pela WEDTT e pelos colaboradores-chave. Entre as recomendações da WEDTT, estão políticas para melhorar a responsabilidade social das faculdades de Medicina dos Estados Unidos, estratégias centradas nos desejos das gerações mais jovens e envolvimento precoce dos jovens com a carreira ${ }^{26}$.

No Brasil, diversas iniciativas têm sido implementadas no sentido de incentivar a formação médica com perfil para a APS, e a mais recente foi o PMM que, além do provimento emergencial de médicos, buscou estimular a formação de profissionais para a APS ${ }^{13}$. De 2013 a 2016, com o início do PMM, observou-se aumento de mais de 1.700 vagas de RMFC, entretanto a ociosidade se manteve em torno dos $70 \%{ }^{27}$. Essa ociosidade de vagas tem se mantido constante desde $2011^{28}$. Mesmo entre os profissionais que trabalham no PMM, muitos não se interessam por seguir carreira na APS, como evidencia um estudo realizado em 2015 nas quatro maiores universidades públicas de Minas Gerais, com residentes recém-ingressantes em todas as áreas de residência médica. $O$ referido estudo identificou que, dos residentes que já haviam trabalhado na APS (grande parte deles em programas de incentivo à APS, como o Programa de Valorização do Profissional da Atenção Básica (Provab) e o PMM), apenas 3,7\% tinham como motivação, ao entrarem nesses programas, seguir carreira na APS. O mesmo estudo revelou que $36 \%$ dos residentes entrevistados cogitaram escolher alguma especialidade da atenção primária ao longo da graduação, dos quais $61 \%$ optaram por essa área depois de formados ${ }^{29}$. Os dados revelam que ainda há uma fragilidade dos mecanismos de incentivo à escolha e fixação dos profissionais médicos na APS. Talvez seja necessário que a esses incentivos sejam incorporadas estratégias relacionadas a planos de carreira, à adequação de estruturas e a mecanismos de apoio remoto a esses profissionais ${ }^{30,31}$.

A associação da intenção de seguir carreira na APS com o sexo feminino está em consonância com outros estudos brasileiros e internacionais ${ }^{18,29,32}$. Um estudo realizado em Minas Gerais identificou que mulheres têm chance 2,9 vezes maior de optar por carreira na APS ${ }^{29}$, resultado muito semelhante ao encontrado por esta pesquisa. Alguns estudos identificam ainda fatores que interferem na escolha de especialidade por mulheres, entre os quais se destacam horas de trabalho, qualidade de vida e tempo para se dedicar à família, além de foco na saúde pública, relação médico-paciente e satisfação pessoal ${ }^{33,34}$.

Também foi identificado como fator associado o ingresso na graduação por meio do Prouni, um programa 
do governo federal, que oferece bolsas de estudo parciais ou integrais em IES privadas para estudantes de baixa renda. Não se identificaram outros estudos que avaliaram ou que tenham identificado a mesma associação, entretanto alguns estudos indicam que a renda familiar está associada à escolha da especialização médica ${ }^{34}$. Um estudo realizado em 2016 na Universidade Estadual de Campinas (Unicamp), logo após mudança no processo de vestibular, com implementação de política de bonificação para estudantes de escolas públicas e autodeclarados negros, aponta mudança na escolha de carreira e especialidade médica, com aumento da intenção de trabalhar somente no SUS após a graduação ${ }^{35}$. Os autores inferem que essa associação também possa estar relacionada à percepção de dever de devolução para a sociedade, pelo fato de terem cursado a graduação por meio de bolsa concedida pelo governo. Porém, são necessários estudos mais específicos para que essa associação possa ser avaliada. A partir dessa associação encontrada, é possível inferir que as políticas de acesso ao ensino superior, como o Prouni, também podem contribuir para a formação de profissionais para as necessidades do SUS.

Os estudantes com graduação anterior revelaram maior interesse em seguir carreira na APS, porém os motivos que os levaram a iniciar um novo curso superior em Medicina são pouco investigados. Entre os fatores que podem influenciar a busca por essa nova formação e que se aproximam da escolha de seguir carreira na APS, estão a busca de maior valorização pessoal e profissional, não alcançada no primeiro curso, e melhores remuneração e empregabilidade. A remuneração e o mercado de trabalho em APS, com disponibilidade de vagas, podem justificar essa associação ${ }^{36}$.

A aptidão autorreferida para especialidades clínicas foi outro fator associado à intenção de carreira na APS. Um estudo internacional evidencia que estudantes que referem maior interesse por raciocínio diagnóstico clínico tendem a optar mais por carreiras na APS ${ }^{20}$. Embora não tenham sido identificados outros estudos com análise similar, a associação com o interesse por áreas clínicas parece ser óbvia, considerando as principais atividades desenvolvidas na APS. Um estudo prévio já apontou que o interesse na APS durante a faculdade aumenta em mais de quatro vezes a chance de optar por essa carreira no final do curso $^{29}$. Considerar a possibilidade de carreira na APS desde o momento da matrícula também está associado a essa opção após a graduação ${ }^{18}$.

A associação de boa experiência na APS durante o currículo com preferência por carreira na APS está em consonância com outros estudos que demonstram a importância tanto de uma experiência positiva na APS quanto de uma maior exposição à MFC durante o currículo, além de experiências com médicos de família na graduação, no incentivo à escolha de carreiras nessa área ${ }^{5,12,25,37}$. Em 2009, uma revisão de literatura sobre o interesse dos estudantes de Medicina pela carreira de médicos de família apontava a pouca vivência em APS durante a graduação, ou ainda a experiência em medicina de família com um profissional desmotivado e frustrado, como um dos motivos para a falta de interesse em seguir essa carreira. As mais recentes DCN do curso de graduação em Medicina, publicadas em 2014, estimularam mudanças curriculares que incluíram a inserção inicial e mais prolongada do estudante na prática da $\mathrm{APS}^{38}$. Assim, corroboram-se as considerações de Cavalcante Neto et al. ${ }^{10}$ de que a experiência exitosa na APS, de fato, pode ser um fator motivador para levar jovens médicos a seguir carreira nessa área.

Ainda que as mudanças curriculares, no que tange à inserção de estudantes na APS desde o início da graduação, reflitam positivamente na escolha de carreira de médicos na APS, a organização dos cursos médicos em metodologias tradicionais ativas de aprendizagem não parece ter impacto na escolha de atuação na APS. Um estudo realizado no Rio de Janeiro, que investigou o impacto das mudanças curriculares com o uso de metodologias ativas, além da inserção inicial do estudante na APS, relacionando com a intenção dos estudantes do quinto período em escolher MFC como especialidade, mostrou que as mudanças no curso são bem avaliadas pelos alunos, entretanto não impactaram a escolha da carreira na APS, que permanece pouco valorizada ${ }^{39}$.

Outros fatores não avaliados neste trabalho são descritos na literatura como influências positivas na intenção de carreira em APS, como: comprometimento e atitudes empáticas ${ }^{25,40}$, interações com pacientes, profissionais da saúde ${ }^{28,41}$ e comunidade $20,41,42$, questões financeiras ${ }^{41,42}$, bom equilíbrio entre vida profissional e pessoal ${ }^{42,43}$, valorização das relações médico-paciente de longo prazo ${ }^{18,28,43}$, prestar cuidados em diferentes fases de vida ${ }^{18,42}$, vivenciar atendimentos em área rural $^{5,18}$ e possibilidade de atuar com medicina preventiva ${ }^{20}$.

Os autores destacam alguns fatores como influências negativas para a escolha da carreira na APS, como o preconceito e estigma percebidos em escolas médicas em relação ao trabalho na APS e a baixa remuneração ${ }^{10,31,42,44}$, condições adversas de trabalho e baixo status social e profissional ${ }^{10,28,31}$.

Os resultados deste estudo devem ser considerados à luz de algumas limitações. Trata-se de um consolidado de uma amostra de conveniência, o que restringe a generalização dos dados. É possível que as instituições selecionadas não representem adequadamente os concluintes de Medicina de todo o país (seja pelas características socioeconômicas ou pelas perspectivas e oportunidades de trabalho, aspectos que podem interferir na escolha do campo de trabalho após a graduação). Todavia, o número de respondentes é expressivo, e os resultados 
são bastante relevantes e não devem ser desconsiderados. O fato de não existir um instrumento padronizado e válido (nacional ou internacionalmente) também pode limitar o processo de comparação dos resultados com outros estudos. Ainda assim, as pesquisas sobre o tema abordam um construto particular, que retrata o interesse do respondente, e indicam aspectos que devem ser considerados na organização de políticas públicas que contribuam para o interesse dos profissionais médicos em atuar em áreas prioritárias para o sistema de saúde.

Assim, entende-se que os resultados deste estudo devem ser considerados por gestores da área da saúde e da educação médica, fomentando novas propostas de formação profissional para o SUS, com maior incentivo à integração ensino-serviço e com inserção, ao longo da graduação, de estudantes em uma rede de APS fortalecida. Algumas estratégias podem ser implementadas a fim de aumentar o número de médicos que desejem atuar na APS, como a ampliação de carga horária na APS durante a graduação, o aumento do número de professores especialistas em MFC e a qualificação da rede de saúde dos municípios onde essas IES estão inseridas. Além disso, iniciativas como plano de carreira, investimento em infraestrutura da rede de saúde e valorização dos profissionais que nela atuam podem contribuir para aumentar esse interesse nos egressos dos cursos de graduação em Medicina. Programas como o PMM têm impacto no provimento emergencial de médicos e na ampliação de graduandos em Medicina, entretanto o que se mostra mais impactante na formação de profissionais com perfil para o SUS e no interesse dos graduandos em atuar na APS são experiências positivas nessa área durante a graduação.

\section{CONCLUSÃO}

Os resultados desta pesquisa evidenciam que mais de um quarto dos estudantes de Medicina das universidades pesquisadas pretende atuar na APS após a conclusão do curso, percentual mais alto do que foi registrado em estudos anteriores, mas ainda aquém das necessidades do SUS. Entre os fatores associados à intenção de atuar na APS, chama a atenção a vivência de uma experiência exitosa na APS ao longo da graduação, pelo fato de ser um fator modificável. Assim, estratégias como ampliação de carga horária na APS durante a graduação, aumento do número de professores especialistas em MFC e qualificação da rede de saúde dos municípios onde essas IES estão inseridas podem ser potentes no sentido de elevar o interesse dos formandos pela atuação na APS.

O ingresso na graduação por meio do Prouni também é um fator associado à intenção de atuar na APS que merece destaque, pois as políticas de acesso ao ensino superior podem contribuir para a formação de profissionais que se dediquem às necessidades doSUS. Considerando que não foram identificados estudos que tenham avaliado a mesma associação, sugere-se que novas pesquisas sejam feitas no sentido de compreender os fatores que interferem nesse resultado.

Embora o estudo não tenha investigado a relação das escolhas dos estudantes com os contextos social, político e econômico do país, é possível que os resultados traduzam, pelo menos parcialmente, uma insegurança por parte dos discentes quanto à carreira na APS. Existem estudos internacionais que investigam as motivações dos estudantes para as escolhas de carreira, porém na literatura encontram-se poucas pesquisas brasileiras nesse sentido. Pelo fato de essa escolha estar relacionada, entre outras questões, com os cenários político e econômico do país, sugere-se também que pesquisas sejam realizadas buscando associações com as escolhas de carreira, no sentido de contribuir para a proposição de novas estratégias que visem solucionar esse problema.

\section{CONTRIBUIÇÃO DOS AUTORES}

Frantchesca Fripp dos Santos, Camila Zamban de Miranda e Karina Cenci Pertile participaram da concepção da pesquisa, da coleta e análise dos dados, da elaboração do artigo e da revisão crítica do texto. Simone de Melo Costa e Antônio Prates Caldeira orientaram todas as etapas da pesquisa e do desenvolvimento do manuscrito, e participaram da coleta e análise dos dados, da elaboração do artigo e da revisão crítica do texto. Mariane Silveira Barbosa participou da coleta e análise dos dados, e da revisão crítica do texto.

\section{CONFLITO DE INTERESSES}

Declaramos não haver conflito de interesses.

\section{FINANCIAMENTO}

Declaramos não haver financiamento.

\section{REFERÊNCIAS}

1. Brasil. Política Nacional de Atenção Básica. Brasília: Ministério da Saúde 2012.

2. Póvoa L, Andrade MV. Distribuição geográfica dos médicos no Brasil: uma análise a partir de um modelo de escolha locacional. Cad Saude Publica. 2006;22(8):1555-64 [access in 12 jun 2016]. Available from: https://www. scielosp.org/scielo.php?pid=S0102-311X2006000800004\&script $=$ sci_ arttext\&tlng=pt.

3. Campos FE, Machado MH, Girardo SN. A fixação de profissionais de saúde em regiões de necessidades. Divulg Saúde Debate. 2009;44:13-24 [access in 12 jun 2016]. Available from: http://www.cnts.org.br/public/arquivos/ Artigo_Campos.pdf.

4. Girardi SN, Carvalho CL, Araújo JF, Farah JM, Wan der Maas L, Campos LAD Índice de escassez de médicos no Brasil: estudo exploratório no âmbito da atenção primária. In: Pierantoni CR, Dal Poz MR, França TO, organizadores. O trabalho em saúde: abordagens quantitativas e qualitativas. Rio de Janeiro: Cepesc, IMS, Uerj-ObservaRH; 2011. p. 171-86.

5. Alavi M, Ho T, Stisher C, Richardson E, Kelly C, McCrory K, et al. Factors that influence student choice in family medicine. Fam Med. 2019;51(2):143-8. 
6. Petterson SM, Liaw WR, Tran C, Bazemore AW. Estimating the residency expansion required to avoid projected primary care physician shortages by 2035. Ann Fam Med. 2015;13(2):107-14

7. Kawamoto R, Ninomiya D, Kasai Y, Kusunoki T, Ohtsuka N, Kumagi T, et al. Gender difference in preference of specialty as a career choice among Japanese medical students. BMC Med Educ. 2016;16(1):288-96.

8. Kirch DG, Henderson MK, Dill MJ. Physician workforce projections in an era of health care reform. Annu Rev Med. 2012;63(1):435-45.

9. CAPER - Canadian Post-M.D. Education Registry. Field of Post-M.D. Training by Faculty of Medicine Providing Post-M.D. Training 2013-2014. 2015;3 [access in 13 jun 2016]. Available from: www.caper.ca.

10. Cavalcante Neto PG, Lira GV, Miranda AS. Interesse dos estudantes pela medicina de família: estado da questão e agenda de pesquisa. Rev Bras Educ Med. 2009;33(2):198-204.

11. Anjos RMP, Gianini RJ, Minari FC, de Luca AHS, Rodrigues MP. “Vivendo o SUS": uma experiência prática no cenário da atenção básica. Rev Bras Educ Med. 2010;34(1):172-83.

12. Campedelli-lopes AM, Bicudo AM, Antonio MARG. A evolução do interesse do estudante de Medicina a respeito da atenção primária no decorrer da graduação. Rev Bras Educ Med. 2016;40(4):621-6.

13. Brasil. Lei $n^{\circ} 12.871$, de 22 de outubro de 2013. Institui o Programa Mais Médicos, altera as Leis no 8.745 , de 9 de dezembro de 1993, e n 6.932 , de 7 de julho de 1981, e dá outras providências. Diário Oficial da União; 23 out 2013.

14. Girardi SN, Stralen ACDSV, Cella JN, Wan Der Maas L, Carvalho CL, Faria EDO. Impacto do Programa Mais Médicos na redução da escassez de médicos em atenção primária à saúde. Cien Saude Colet. 2016;21(9):2675-84.

15. Pfarrwaller E, Sommer J, Chung C, Maisonneuve H, Nendaz M, Perron $\mathrm{NJ}$, et al. Impact of interventions to increase the proportion of medical students choosing a primary care career: a systematic review. J Gen Intern Med. 2015;30(9):1349-58.

16. Mello GA, Mattos ATRD, Souto BGA, Fontanella BJB, Demarzo MMP. Médico de família: ser ou não ser? Dilemas envolvidos na escolha desta carreira. Rev Bras Educ Med. 2009;33(3):475-82.

17. Kost A, Bentley A, Phillips J, Kelly C, Prunuske J, Morley C. Graduating medical student perspectives on factors influencing specialty choice. Fam Med. 2019;51(2):129-36.

18. Deutsch T, Lippmann S, Frese T, Sandholzer H. Who wants to become a general practitioner? Student and curriculum factors associated with choosing a GP career - a multivariable analysis with particular consideration of practice-orientated GP courses. Scand J Prim Health Care. 2015;33(1):47-53.

19. Lefevre JH, Roupret $M$, Kerneis $S$, Karila L. Career choices of medical students: a national survey of 1780 students. Med Educ. 2010,44(6):603-12.

20. Bilal M, Haseeb A, Mari A, Arshad MH, Khan MRA, Ahmed A, et al. Factors determining Pakistani medical students' career preference for general practice residency training. Cureus. 2018;10(8):e3114.

21. Eggertson L. More medical graduates than ever choosing family practice. Can Med Assoc J. 2015;187(9):644.

22. Mirvis DM. Choosing a medical specialty: the difference between what students want and what society needs. Isr J Health Policy Res. 2013;2(1):18-25.

23. Svirko E, Goldacre MJ, Lambert T. Career choices of the United Kingdom medical graduates of 2005, 2008 and 2009: questionnaire surveys. Med Teach. 2013;35(5):365-75.

24. Oliveira NA, Alves LA. Ensino médico, SUS e início da profissão: como se sente quem está se formando? Rev Bras Educ Med. 2011;35(1):26-36.

25. Souza LCL, Mendonça VR, Garcia GB, Brandão EC, Barral-Netto M. Medical specialty choice and related factors of Brazilian medical students and recent doctors. PLoS One. 2015;10(7):10-8.

26. Kelly C, Coutinho A, Goldgar C, Gonsalves W, Gutkin C, Kellerman R, et al. Collaborating to achieve the optimal family medicine workforce. Fam Med. 2019;51(2):149-58.
27. Trindade TG, Batista SR. Medicina de Família e Comunidade: agora mais do que nunca! Cien Saude Colet. 2016;21(9):2667-9.

28. Rolim TV, Queiroz OS, Monteiro ABC, Asfor ATP, Sousa TS. Especialização em Medicina de Família e Comunidade: visão dos acadêmicos de Sobral. Anais do $12^{\circ}$ Congresso Brasileiro Medicina Família Comunidade; 2013; Belém, Brasil. Belém: Sociedade Brasileira de Medicina de Família e Comunidade; 2013.

29. Oliveira PRBP. A escolha da especialidade por ingressantes na residência médica do estado de Minas Gerais [dissertação]. Campinas: Universidade Estadual de Campinas; 2015.

30. Ney MS, Rodrigues PHA. Fatores críticos para a fixação do médico na Estratégia Saúde da Família. Physis. 2012;22(4):1293-1311 [access in 14 jun 2017]. Available from: https://www.scielosp.org/scielo.php?pid=S0103$73312012000400003 \&$ script=sci_arttext\&tlng=en.

31. Tinoco AS, Oliveira IC, Cutolo LRA, Maeyama MA. Percepção dos estudantes de medicina acerca da Residência em Medicina de Família e Comunidade. Revista Brasileira de Tecnologias Sociais. 2017;4(1):75-87.

32. Kiolbassa K, Miksch A, Hermann K, Loh A, Szecsenyi J, Joos S, et al. Becoming a general practitioner: which factors have most impact on career choice of medical students? BMC Fam Pract. 2011;12(1):327-45.

33. Corsi PR, Fernandes EL, Intelizano PM, Montagnini CCB, Baracat FI, Ribeiro MCSA. Fatores que influenciam o aluno na escolha da especialidade médica. Rev Bras Educ Med. 2014;38(2):213-20 [access in 7 sep 2018] Available from: https://www.researchgate.net/profile/Paulo_Corsi/ publication/316004128 Fatores que influenciam_o aluno na escolha da_especialidade_medica/links/5946e9c20f7e9b6910f72b4d/Fatores-queinfluenciam-o-aluno-na-escolha-da-especialidade-medica.pdf.

34. Sousa IQ, Silva CP, Caldas CAM. Especialidade médica: escolhas e influências. Rev Bras Educ Med. 2014;38(1):79-86.

35. Silva MLADM, Amaral E, Machado HDC, Passeri SMRR, Bragança JF. Influência de políticas de ação afirmativa no perfil sociodemográfico de estudantes de Medicina de universidade brasileira. Rev Bras Educ Med. 2018;42(3):36-48

36. Corrêa RD, Gonçalves RCB, Oliveira LSD, Silva VCM, Ribeiro MMF. Medicina como nova graduação: motivações, dificuldades e expectativas. Rev Bras Educ Med. 2016;40(2):226-33.

37. Issa AHTM, Garcia-Zapata MTA, Rocha CA, Sandré BB, Dutra ACF, Martin ILO, et al. Fatores influenciadores na escolha pela medicina de família segundo estudantes numa região neotropical do Brasil. Rev Educ Saúde. 2017;5(2):56-65.

38. Brasil. Parecer CES/CNE n 116/2014. Diário Oficial da União; 6 jun 2014 Seção 1, p. 17

39. Costa JRB, Romano VF, Costa RR, Gomes AP, Alves LA, Batista RS. A transformação curricular e a escolha da especialidade médica. Rev Bras Educ Med. 2014;38(1):47-58.

40. Ster M, Selic P. Intended career choice in family medicine in Slovenia: an issue of gender, family background or empathic attitudes in final year medical students? Mater Sociomed. 2017;29(2):143-8.

41. Weiland G, Cox K, Sweeney MK, Belue M, Snyder ED, Curry WD, et al. What attracts medical students to primary care? A nominal group evaluation. South Med J. 2019;112(2):76-82.

42. Merrett A, Jones D, Sein K, Green T, Macleod U. Attitudes of newly qualified doctors towards a career in general practice: a qualitative focus group study. Br J Gen Pract. 2017;67(657):253-9.

43. Osborn HA, Glicksman JT, Brandt MG, Doyle PC, Fung, K. Primary care specialty career choice among Canadian medical students: understanding the factors that influence their decisions. Can Fam Physician. 2017;63:107-13.

44. Magalhães TN, Belmonte TSA, Luna CAA. Medicina de família na educação médica: um núcleo de ensino na atenção terciária para a aprendizagem em atenção primária. Cad Bras Med. 2014;27(3):1-58.

This is an Open Access article distributed under the terms of the Creative Commons Attribution License, which permits unrestricted use, distribution, and reproduction in any medium, provided the original work is properly cited. 